\title{
LATE RESPONSES IN THE ELECTRODIAGNOSIS OF CERVICAL RADICULOPATHIES
}

\author{
ANA MARIA GALAMB, IOAN DAN MINEA
}

\author{
Department of Medical and Surgical Specialities, Faculty of Medicine, Transilvania \\ University of Brasov, Romania
}

\begin{abstract}
Backround and aim. This paper aims to assess the parameters of late responses and then determine their usefulness in patients with cervical radiculopathy.

Patients and methods. We studied a total of 114 patients with bilateral assessment of median and ulnar nerves and of $F$ and $A$ waves parameters.

Results. We draw attention to the need of bilateral electrodiagnostic examination due to changes occurring in a third of cases also in the asymptomatic limb. Pluriradicular injuries occurred in one third of cases. The root that was most commonly affected in cases of uniradicular lesion was C7. The parameters with the most important alterations were persistence, tacheodispersion and chronodispersion, which were changed in about a half of the patients. With regard to the A wave, it occurred in a small number of cases, about 10\%, with an average amplitude of about $120 \mu \mathrm{V}$ and an average latency of $15 \mathrm{~ms}$. The distance from the point of stimulation where the collateral branch appeared was calculated to be approximately $35 \mathrm{~cm}$.

Conclusions. Among the $F$ wave parameters, persistence, tacheodispersion and chronodispersion are recommended to be studied; these parameters were also included in the composite score along with the AAEM recommendations.
\end{abstract}

Keywords: cervical radiculopathies, composite score, late responses.

\section{Introduction}

For patients with cervical radiculopathy, electrodiagnostic examination is complementary to imaging studies, a great importance having the detection of axonal injury. EMG is considered positive for the presence of radiculopathy if abnormalities occur in two muscles innervated by the same nerve root, but by different nerves, and also when the muscles that are innervated by another root are without modification, thus eliminating the diagnosis of mononeuropathies and polyneuropathies [1].

The proximal segment of the peripheral nerve is not assessed by the usual electrodiagnostic studies, these areas being able to be investigated only by assessing late responses [1,2].

The principle that underlies the obtainment of late

Manuscript received: 17.12.2014

Accepted: 09.01.2015

Address for correspondence: anagalamb@gmail.com responses is to excite a nerve in its distal portion and obtain a response using surface electrodes, after the excitation propagates proximally to the spinal cord and then returning to the periphery $[2,3]$.

Regarding the $\mathrm{F}$-wave, there are some parameters that are usually evaluated and are recommended to be studied in most specialty literature reports. They are: average latency of F-wave, persistence, presence of repetitive or increased amplitude F-waves. Parameters that are rarely evaluated are: chronodispersion, proximal velocity, tacheodispersion, the ratio between $\mathrm{F}$ and $\mathrm{M}$-wave amplitude. It would be desired to enter into evaluation also the latency difference between $\mathrm{F}$ and $\mathrm{M}$-waves and the $\mathrm{F}$ ratio.

Regarding the A-wave there are no recommendations for its parameters, they should only be mentioned as being present. Given that its presence indicates reinnervation, calculating its parameters: amplitude, latency and latency difference to $\mathrm{F}$-wave may provide information on this process. One can also determine the distance at which 
the collateral branch occurs in relation to the point of stimulation.

\section{Objective}

This paper aims to assess the parameters of late responses and ultimately determine the usefulness of each, taking into study a large number of cases and investigating even the least used parameters.

\section{Material and method}

We included a total of 114 patients with mean age 52 and the female/male ratio 55.26/44.74.

The examination included the bilateral study of the median, ulnar and radial nerves, including the assessment of F and A-waves' parameters. Also electromyography of the deltoideus, triceps brachii and abductor pollicis brevis was performed. When appropriate, the number of nerves and muscles was expanded for an accurate diagnosis.

The investigation method was approved by the Ethical Committee of the Faculty of Medicine - Transilvania University of Brasov. The study was accomplished according to the WMA Declaration of Helsinki. All patients were given a detailed explanation of the study and all provided a written informed consent.

Statistical analysis was performed using SPSS 13.0 for Windows.

The parameters of the late responses, having the highest observed percentage of changes, will lead to the formulation of a composite score, along with the recommendations of the American Association of Electrodiagnostic Medicine (AAEM) for cervical radiculopathies [9].

\section{Technical examination}

The F-wave was defined as the second wave occurring after the $\mathrm{M}$ response with minimum amplitude of $100 \mu \mathrm{V}$ at supramaximal stimulation of the motor nerve, recording on the muscle innervated by that nerve. A third wave occurring between $\mathrm{M}$ and $\mathrm{F}$-wave response, is defined as A-wave, being differentiated from the F-wave by constant morphology, latency and amplitude.

For the median nerve stimulation was applied at the wrist, between the tendons of flexor carpi radialis and palmaris longus, with the cathode oriented proximally. The recording was made with surface electrodes, the active one applied on the muscle belly of abductor pollicis brevis and the reference distal to it at least $3 \mathrm{~cm}$, the examined muscle being relaxed. The ground electrode was placed on the dorsal aspect of the hand. The skin was cleaned with alcohol before placing electrodes.

For the ulnar nerve stimulation was applied at the wrist medial to the flexor carpi ulnaris tendon, with the cathode oriented proximally. The recording was made with surface electrodes, the active one applied on the muscle belly of abductor digiti minimi and the reference distal to it at least $3 \mathrm{~cm}$, the examined muscle being relaxed. The ground electrode was placed on the dorsal aspect of the hand. The skin was cleaned with alcohol before placing electrodes.

To determine the supramaximal current intensity that needs to be applied, the same assembly of electrodes was used, but with the cathode oriented distally and the current intensity was gradually increased to obtain maximum amplitude of the $\mathrm{M}$ response. To obtain F-waves, a current $30 \%$ higher than the above was used.

Each nerve was stimulated with the respective intensity of the current with a duration of $0.2 \mathrm{~ms}$, using 10 to 20 consecutive stimulations at a frequency of $1 \mathrm{~Hz}$.

The surface temperature of the limbs was 32 degrees Celsius.

Screen settings for each examination were $7.5 \mathrm{~ms} /$ div, $1.5 \mathrm{mV} / \mathrm{div}$, low cut filter $5 \mathrm{~Hz}$ and high cut 10,000 Hz.

\section{Studied parameters}

The studied parameters for F-wave were:

1. The minimum latency;

2. The average latency;

3. The maximum latency;

4. Chronodispersion (defined as the difference between the minimum and maximum latency);

5. The difference between the minimum latency of F-wave and the latency of M-wave;

6. The difference between the average latency of F-wave and the latency of M-wave;

7. The difference between the maximum latency of wave F-wave and the latency of M-wave;

8. Proximal maximum velocity calculated by the formula: (the distance from the point of stimulation and C7) x2 / ((latency F-latency M) - $1 \mathrm{msec})$;

9. Proximal average velocity calculated by the formula: maximum proximal velocity $\mathrm{x}$ ((minimum latency of F-1) / (average latency of F-1));

10. Minimum proximal velocity calculated by the formula: maximum proximal velocity $\mathrm{x}$ ((minimum latency of F-1) / (maximum latency of F-1));

11. tacheodispersion (defined as the difference between the maximum and minimum velocity);

12. The average ratio of the amplitudes F/M;

13. The maximum ratio of the amplitudes $\mathrm{F} / \mathrm{M}$;

14. The percentage of F-waves with high amplitude (greater than $1 \mathrm{mV}$ );

15. F-wave persistence;

16. The percentage of repetitive F-waves;

17. F ratio calculated using the formula: ((latency F-latency M) -1ms) / (latency Mx2);

18. The right-left difference between the mean latencies of the F-wave of the median nerve;

19. The right-left difference between average velocity of the F-wave of the median nerve. 
All this was calculated bilaterally for the median and ulnar nerves.

The studied parameters for A-wave were:

1. The amplitude;

2. The latency;

3. The latency difference between A-wave and F-wave;

4. Calculation of the distance from the generating branch to the point of stimulation.

M-wave, F-wave and A-wave latencies were calculated from the deflexion of those waves and the amplitudes were calculated peak-to-peak [4].

\section{Results}

$56.82 \%$ of all patients had a diagnosis of suspected cervical injury. Electrodiagnosis revealed a bilateral damage in $49.12 \%$ of the cases. EMG diagnosis showed pluriradicular injury in $42.11 \%$ of the cases. In cases with severe uniradicular root, C7 was the most frequently affected in $42.98 \%$ of patients, C5 in $7.89 \%$, and C8-T1 in $6.14 \%$.

$17.54 \%$ of cases had also entrapment neuropathy of the median nerve in the carpal tunnel.

In terms of F-waves of the median and ulnar nerves,

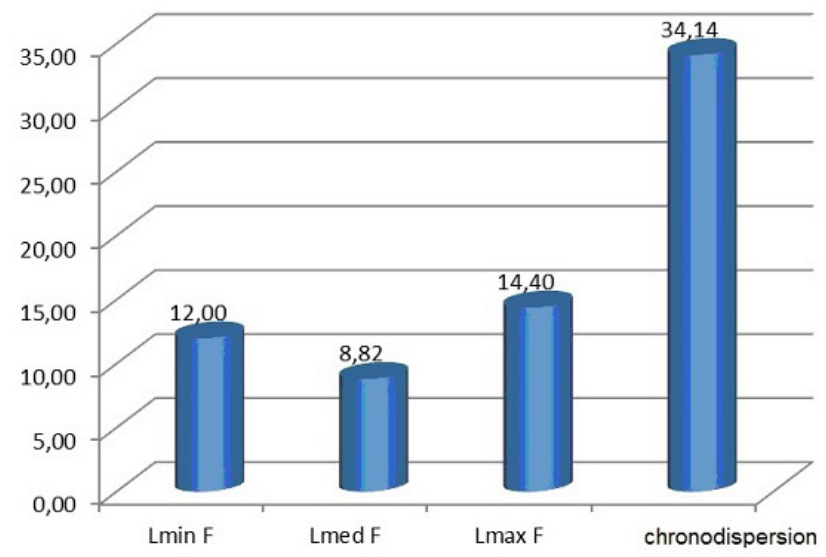

Figure 1. F-wave latencies and choronodispersion, expressed as a percentage, for the upper limbs.

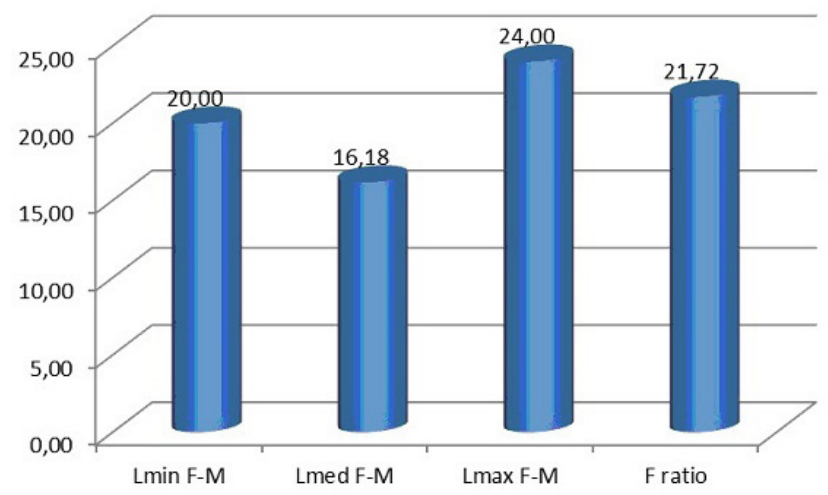

Figure 2. Latency differences between F-wave and M-wave; F ratio, expressed as a percentage, for the upper limbs. the minimum latency of this wave was changed in $12 \%$, average latency in $8.82 \%$ and maximum in $14.40 \%$ of cases. Chronodispersion showed changes in $34.14 \%$ of patients (Fig. 1).

The minimum latency difference between the $\mathrm{F}$ and M-wave showed changes in $20 \%$, the average in $16.18 \%$ and the maximum in $24 \%$ of the cases. F ratio had values lower than 0.7 in $21.72 \%$ of patients (Fig. 2).

F-waves of increased amplitude where present in $27.38 \%$ of cases, persistence was altered in $46.03 \%$, and $9.52 \%$ of patients had an increased number of repetitive

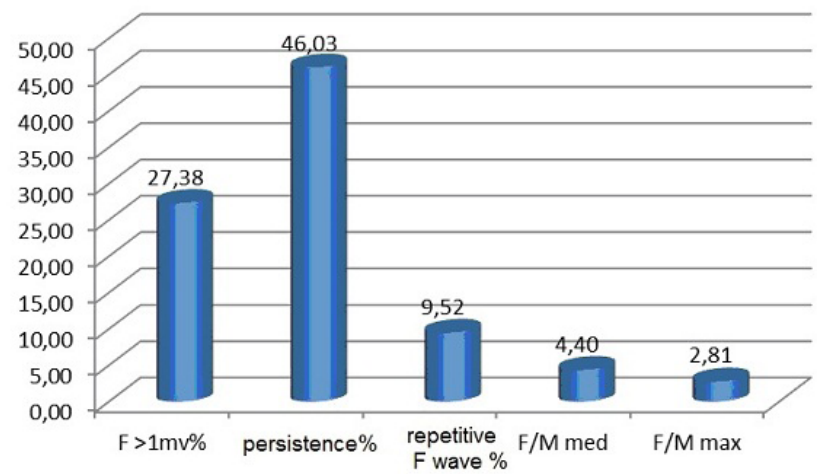

Figure 3. F-wave parameters, expressed as a percentage, for the upper limbs (continuation).

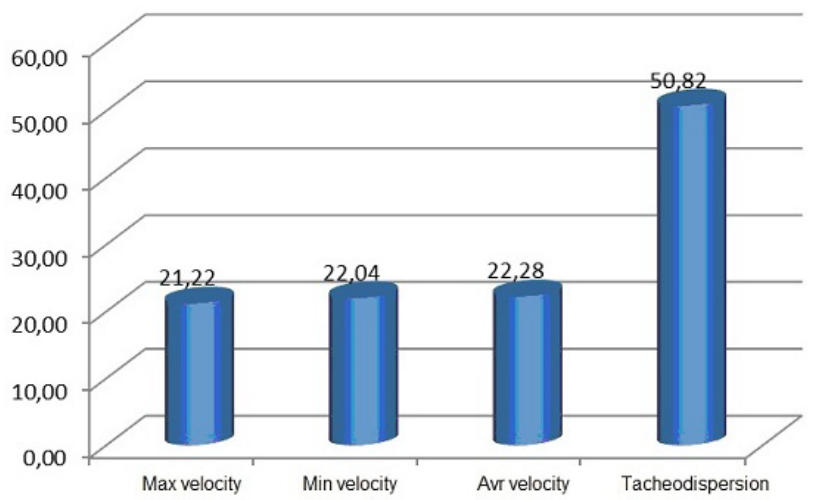

Figure 4. F-wave velocities and tacheodispersion, expressed as a percentage, for the upper limbs.

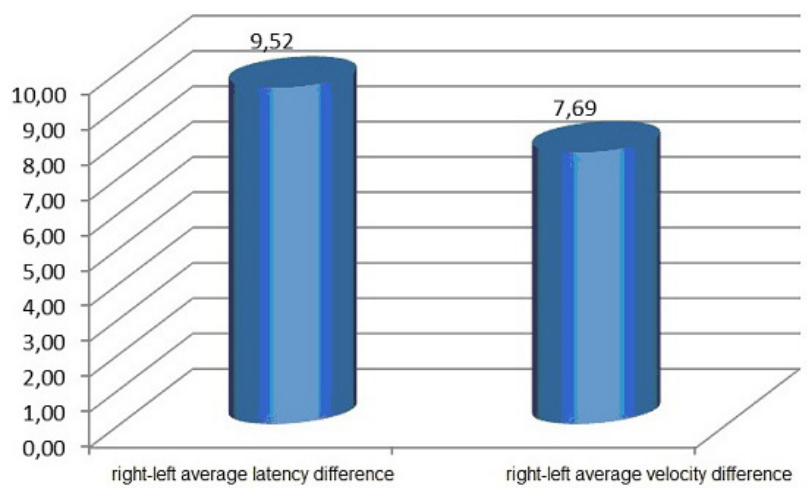

Figure 5. Right-left differences of latency and average velocity of the F-waves. 
Table I. Pearson correlations between F-wave parameters (set I).

\begin{tabular}{|c|c|c|c|c|c|c|c|}
\hline & $\begin{array}{c}\mathrm{M} \\
\text { latency }\end{array}$ & $\begin{array}{l}\text { Min F } \\
\text { latency }\end{array}$ & $\begin{array}{c}\text { Avr F } \\
\text { latency }\end{array}$ & $\begin{array}{l}\text { Max F } \\
\text { latency }\end{array}$ & $\begin{array}{l}\text { Min F-M } \\
\text { latency }\end{array}$ & $\begin{array}{l}\text { Avr F-M } \\
\text { latency }\end{array}$ & $\begin{array}{c}\text { Max F-M } \\
\text { latency }\end{array}$ \\
\hline Min F latency & $\begin{array}{c}r=.613 * * \\
p=.001\end{array}$ & N/A & N/A & N/A & N/A & N/A & N/A \\
\hline Avr F latency & $\begin{array}{c}\mathrm{r}=.518 * * \\
\mathrm{p}=.023\end{array}$ & N/A & N/A & N/A & N/A & N/A & N/A \\
\hline Max F latency & $\begin{array}{c}\mathrm{r}=.479 * * \\
\mathrm{p}=.038\end{array}$ & N/A & N/A & N/A & N/A & N/A & N/A \\
\hline Min F-M latency & N/A & $\mathrm{N} / \mathrm{A}$ & N/A & N/A & N/A & N/A & N/A \\
\hline Avr F-M latency & N/A & N/A & N/A & N/A & N/A & N/A & N/A \\
\hline chronodispersion & $\begin{array}{l}\mathrm{r}=.041 \\
\mathrm{p}=.850\end{array}$ & $\begin{array}{l}\mathrm{r}=-.148 \\
\mathrm{p}=.489\end{array}$ & $\begin{array}{l}r=.348 \\
p=.096\end{array}$ & $\begin{array}{c}r=.698 * * \\
p=.001\end{array}$ & $\begin{array}{c}r=.558 * * \\
p=.002\end{array}$ & $\begin{array}{c}\mathrm{r}=.654 * * \\
\mathrm{p}=.000\end{array}$ & $\begin{array}{c}\mathbf{r}=.709 * * \\
\mathbf{p}=.001\end{array}$ \\
\hline
\end{tabular}
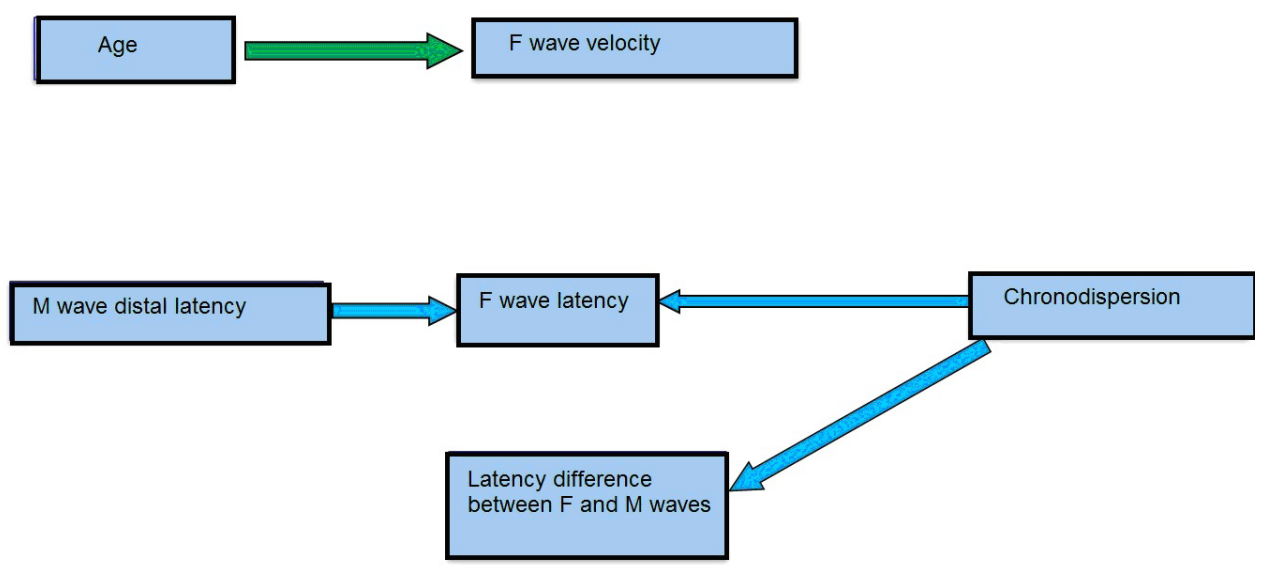

Figure 6. Pearson correlations related to age, M-wave distal latency and chronodispersion.

F-waves. The average amplitude ratio between $\mathrm{F}$ and M-waves was altered in $4.40 \%$ and the maximum in $2.81 \%$ of cases (Fig. 3).

Maximum velocity was modified in $21.22 \%$ of patients, the minimum in $22.04 \%$ and the average in $22.28 \%$ of cases. Tacheodispersion presented abnormal values in $50.82 \%$ of cases (Fig. 4).

Right-left difference of the mean latency of the F-waves was altered in $9.52 \%$ of patients, and the leftright difference of the average velocity in $7.69 \%$ of cases (Fig. 5).

Analyzing the correlations in the table above we can see that there is a highly significant positive correlation between the distal latency of the motor response and the minimum, average and maximum latency of the F-wave. However, there is a significant positive correlation between chronodispersion and the minimum, average and maximum latency difference between $\mathrm{F}$ and $\mathrm{M}$-waves, and the maximum latency of the F-waves (Tab. I, Fig. 6).

The patient age was significantly negatively correlated with the minimum, medium and maximum proximal velocity, in the same way appearing the correlations between the velocities and $\mathrm{F}$ ratio. Tacheodispersion showed a significant positive correlation with the minimum, average and maximum proximal velocity and also with the presence of high amplitude $F$ waves. On the other hand, the presence of repetitive F-waves showed a highly significant positive correlation with the average and maximum amplitude ratio between $\mathrm{F}$ and $\mathrm{M}$-waves. There was also a negative correlation between the presence of F-waves blocks and the maximum velocity or maximum ratio between $\mathrm{F}$ and $\mathrm{M}$-waves amplitude (Tab. II, Fig. 7).

The A-waves occurred in only $11.40 \%$ of patients, with a mean amplitude of $120.53 \mu \mathrm{V}$ (Fig. 8), and an average latency of $15.45 \mathrm{~ms}$.

The average distance where the generating branch occurred from the point of stimulation was $37.76 \mathrm{~cm}$ (Fig. 9).

\section{Cervical radiculopathy - composite score:}

In addition to the recommendations of the AAEM in the examination of the patients with cervical radiculopathy [9]:

- At least an examination of the sensory and motor 
response in the affected limb. If more than one of these tests is abnormal, will evaluate other nerves of the same limb or contralateral limb.

- At least one EMG examination for muscles innervated by the roots $\mathrm{C} 5, \mathrm{C} 6, \mathrm{C} 7, \mathrm{C} 8$ and $\mathrm{T} 1$ in the symptomatic limb and also of the cervical paraspinal muscles (except in patients who have undergone laminectomy at this level). Examination will be performed in muscles innervated by the nerve root suspected, but from different nerves; examination will demonstrate normal muscles innervated by proximal and distal roots to the affected root.

We recommend also the study of the following parameters of the F-waves:

- Chronodispersion,

- Tacheodispersion,

Table II. Pearson correlations between F-wave parameters (set II).

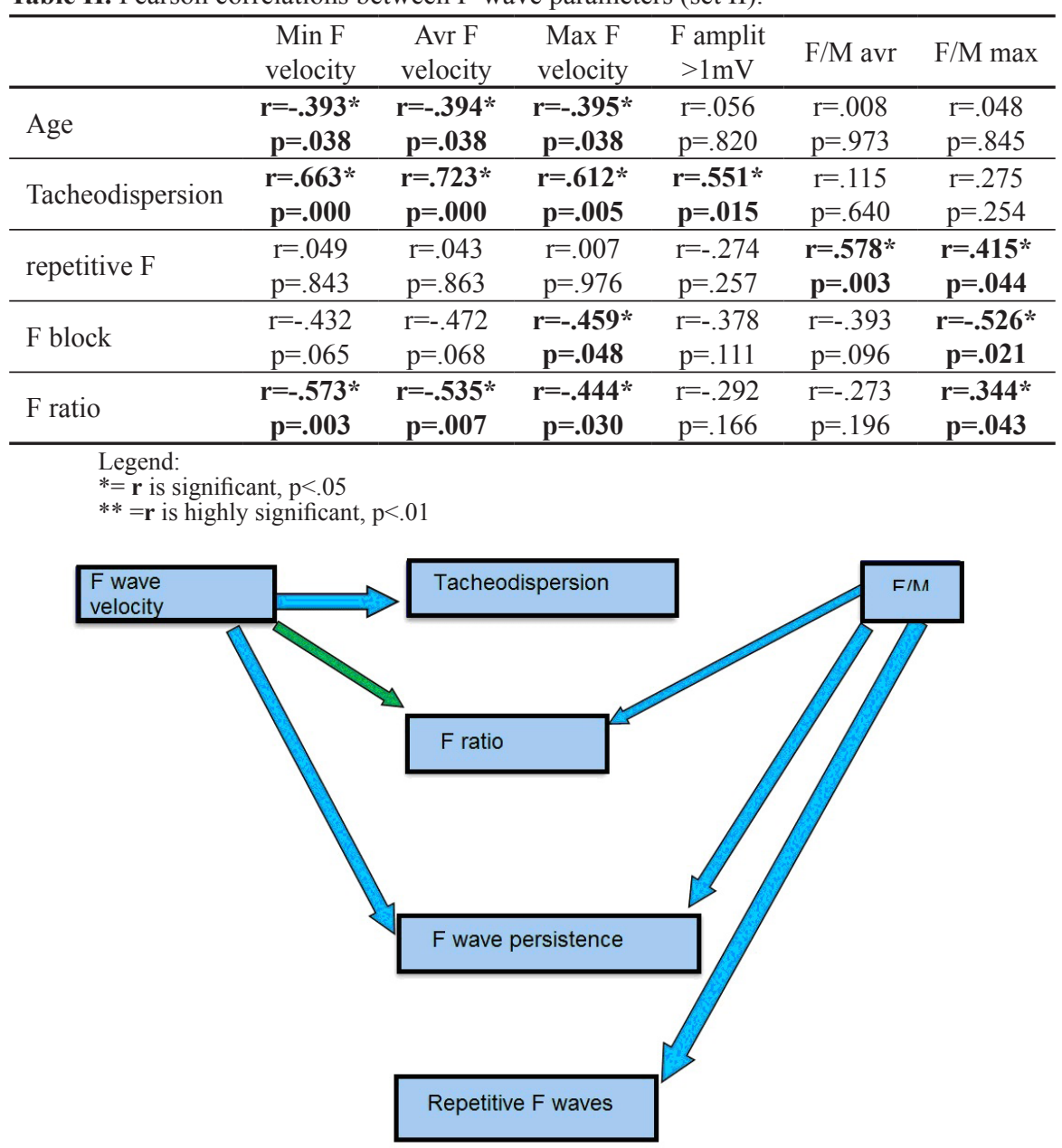

Figure 7. Pearson correlations related to F-wave velocity and F/M ratio.

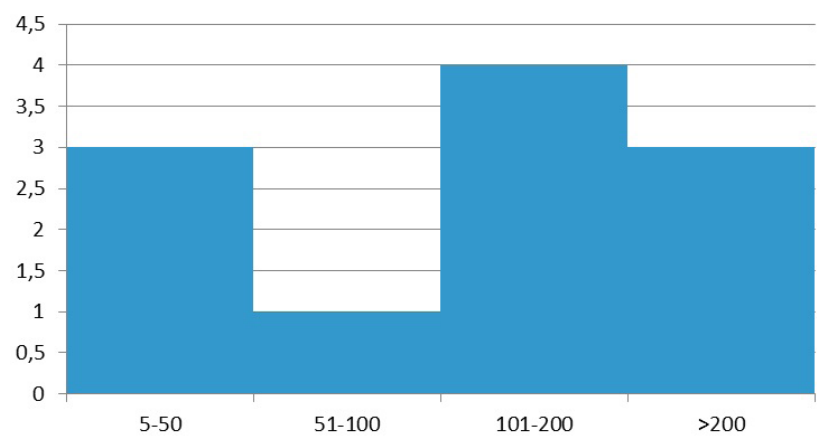

Figure 8. A-wave amplitude $(\mu \mathrm{V})$.

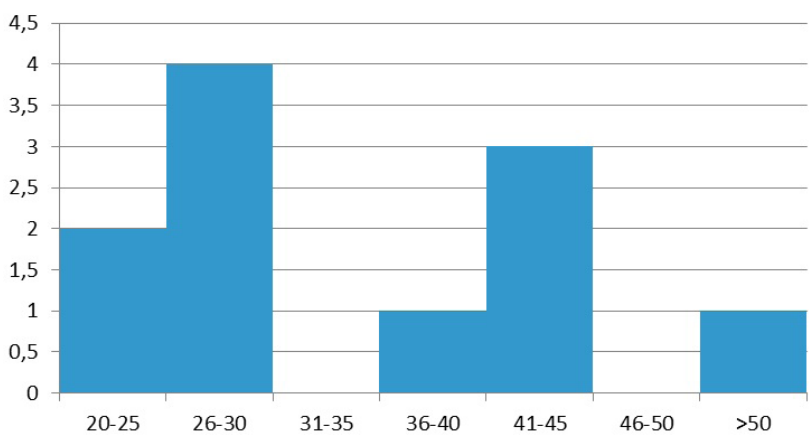

Figure 9. Distance where the generating branch occurred from the point of stimulation $(\mathrm{cm})$. 


\section{- Persistence.}

\section{Discussion and conclusions}

There are different opinions in literature regarding the role of late responses in evaluating patients with radiculopathy, many more studies focusing on the evaluation of lumbar radiculopathy. Ahdab, Dillingham et al. $[5,6]$ conclude that they are nonspecific or that they only serve to exclude the presence of other pathologies.

We reinforce the need for bilateral examination due to an increased number of cases with severe right and left root injury, which was detected in half of the cases in our study.

Pluriradicular injuries occurred in one third of cases. The root that was most commonly affected in cases of uniradicular lesion was C7.

Of the patients examined more than a tenth showed entrapment neuropathy of the median nerve in the carpal tunnel.

From the F-wave parameters, the latencies had changed at a rate less than a quarter of the total cases, slightly more altered were the differences in latency between the $\mathrm{F}$ and $\mathrm{M}$-waves and $\mathrm{F}$ ratio.

Although there are studies that claim that right-left difference in latency of the F-wave is a sensitive parameter for root damage, such as the study by Takahashi et al. [7], the present study revealed changes in only $8 \%$ of cases. These values, however, support the results of Kueter et al. [8], who found also a small percentage of changes in cervical radiculopathy.

Proximal velocity showed alterations in a percentage smaller than a quarter of cases.

The parameters with the most important alterations were persistence, tacheodispersion and chronodispersion, which changed in about a half of the patients.

The very small percentage of the changes observed for these parameters can be due to the fact that the median and the ulnar nerves account only for the lower cervical roots.

With regard to the A-wave, it occurred in a small number of cases, about $10 \%$, with an average amplitude of about $120 \mu \mathrm{V}$, and an average latency of $15 \mathrm{~ms}$. The distance from the point of stimulation where the collateral branch appeared, was calculated to be approximately 35 $\mathrm{cm}$.

Positive correlations were noted between the distal latency of the motor response and F-wave latencies. So, if the first one is prolonged, then we would expect the latter one to increase. Another positive correlation appeared between $\mathrm{F}$-wave latencies or chronodispersion and latency differences between $\mathrm{F}$ and $\mathrm{M}$-waves, so we can conclude that an increase of F-wave latencies will attract an increase in chronodispersion. F-wave velocity was positively correlated with the tacheodispersion and negatively with the presence of F-wave blocks and $\mathrm{F}$ ratio. Thus, the decrease in the velocity can be associated with a lower persistence of these waves. Another positive correlation appeared between the amplitude ratio of $\mathrm{F}$ and $\mathrm{M}$-waves with the presence of repetitive F-waves, suggesting that an increase in F-wave amplitude is associated with an increase in the percentage of F-waves with the same morphology.

\section{References:}

1. Tan FC. EMG Secrets. Philadelphia: Hanley\&Belfus; 2004.

2. Fournier E. Examen electromyographique. $2^{\text {nd }}$ edition. Paris: Edition médicales Internationales; 2008.

3. Nikolaev S. Атлас по электро-миографии (Atlas of electromyography). Ivanovo. PresSto; 2010.

4. Galamb AM, Minea ID, Suiaga D. Cervical Myelopathy vs Amyotrophic lateral sclerosis - the role of electrodiagnostic study. Conferinţa Naţională ASNER. 2013 oct; Abstract book:18. 5. Ahdab R, Lefaucheur JP. Bilan électrophysiologique d'un syndrome du canal lombaire étroit. Pelvi-périnéologie. 2010;5(3):155-163.

6. Dillingham RT. Electrodiagnostic approach to patients with suspected radiculopathy. Phys Med Rehabil Clin N Am. 2002;13:567-588.

7. Takahashi H, Strashill M, Küter L. Value of the F-wave in the Diagnosis of Cervical and Lumbosacral Root Compression Syndromes. Adv Neurosurg. 1978;5:382-388.

8. Takahashi H, Straschill M, Küter L. The value of F-wave in the diagnosis of cervical and lumbo sacral root compression syndromes. Neurol Med Chir (Tokio). 1981;21(4):407-412.

9. American Association of Electrodiagnostic Medicine. Practice parameter for needle electromyographic evaluation of patients with suspected cervical radiculopathy: summary statement. Muscle Nerve. 1999; 22(Suppl 8):S209-S211. 